\title{
Stability analysis of black holes via a catastrophe theory and black hole thermodynamics in generalized theories of gravity
}

\author{
$\operatorname{AUTHOR}(S):$ \\ Tamaki, T; Torii, T; Maeda, K
}

\section{CITATION:}

Tamaki, T ...[et al]. Stability analysis of black holes via a catastrophe theory and black hole thermodynamics in generalized theories of gravity. PHYSICAL REVIEW D 2003, 68(2): 024028.

ISSUE DATE:

2003-07-15

URL:

http://hdl.handle.net/2433/49868

RIGHT:

Copyright 2003 American Physical Society 
PHYSICAL REVIEW D 68, 024028 (2003)

\title{
Stability analysis of black holes via a catastrophe theory and black hole thermodynamics in generalized theories of gravity
}

\author{
Takashi Tamaki* \\ Department of Physics, Kyoto University, Kyoto 606-8502, Japan \\ Takashi Torii ${ }^{\dagger}$ \\ Advanced Research Institute for Science and Engineering, Waseda University, Shinjuku-ku, Tokyo 169-8555, Japan \\ Kei-ichi Maeda \\ Department of Physics, Waseda University, Shinjuku-ku, Tokyo 169-8555, Japan
}

(Received 19 March 2003; published 29 July 2003)

\begin{abstract}
We perform a linear perturbation analysis for black hole solutions with a "massive" Yang-Mills field (the Proca field) in Brans-Dicke theory and find that the results are quite consistent with those via catastrophe theory where thermodynamic variables play an intrinsic role. Based on this observation, we show the general relation between these two methods in generalized theories of gravity which are conformally related to the Einstein-Hilbert action.
\end{abstract}

DOI: 10.1103/PhysRevD.68.024028

PACS number(s): 04.70.-s, 04.40.-b, 95.30.Tg, 97.60.Lf

\section{INTRODUCTION}

Black holes and their thermodynamics are one of the main topics of superstring theory, in particular, following the discoveries of the microscopic origin of the black hole entropy [1] and the AdS/conformal field theory (CFT) correspondence [2]. To consider unified theory including gravity, their importance may be equal to the blackbody radiation which was a clue to developing quantum mechanics.

Recently, the classical stability of $p$-branes and its equivalence to local thermodynamic stability has been argued in Refs. $[3,4]$. Here, thermodynamic stable means that the Hessian of the entropy with respect to the other thermodynamic variables are negative definite in the microcanonical ensemble. Since this equivalence does not hold for black holes (e.g., Schwarzschild black hole), we need another criterion of black holes. In general relativity, we investigated many black hole solutions with non-Abelian matter fields in asymptotically flat space-time and found that stability criterion via catastrophe theory by using thermodynamic variables coincides with those of linear perturbation [5]. A similar discussion in AdS space-time can be seen in Ref. [6]. Iyer and Wald formulated the black hole thermodynamics in arbitrary diffeomorphism invariant theories of gravity [7]. In their formulation, the black hole entropy is defined as a Noether charge and satisfies the first law of black hole thermodynamics. It remains, however, unclear whether or not it satisfies the second law, although some examples were shown in Ref. [8].

Here, we show the relation between linear perturbation analysis and catastrophe theory in generalized theories of gravity which are conformally related to the Einstein-Hilbert action. In the analysis by using catastrophe theory, it is im-

\footnotetext{
*Email address: tamaki@phys.h.kyoto-u.ac.jp

†Email address: torii@gravity.phys.waseda.ac.jp

‡Email address: maeda@gravity.phys.waseda.ac.jp
}

portant which variables we choose as catastrophe variables such as control parameters and a potential function [9]. In general relativity, the mass of the black hole is determined unambiguously in an asymptotically flat space-time and its horizon radius is related geometrically to the entropy. Hence we can choose the natural catastrophe variables. In generalized theories of gravity, however, there are some kinds of the mass, and horizon radius may not be related directly to entropy. There may be other variables depending on theories. Hence there are ambiguities for the choice of the catastrophe variables.

In Sec. II, we review a black hole solution in Brans-Dicke (BD) theory [10] obtained previously as an example [11], and examine its stability by the linear perturbation analysis to confirm the expectation obtained by catastrophe theory. In Sec. III, we discuss what are the suitable variables for the catastrophe analysis in generalized theories of gravity. Next, we show that we can obtain the consistent results with the linear perturbation analysis by adopting the thermodynamic variables defined in Ref. [7] as catastrophe variables in Sec. IV. This leads to the conjecture that the dynamical stability can be judged via catastrophe theory by using thermodynamic variables at least at the linear level. In Sec. V, we offer concluding remarks and discuss future perspectives. Throughout this paper we use units $c=\hbar=G=1$.

\section{PROCA BLACK HOLE IN BRANS-DICKE THEORY AND ITS LINEAR STABILITY}

We briefly review the Proca black hole in BD theory obtained in Ref. [11] and perform a linear perturbation analysis as an example to obtain the clue to the general case.

\section{A. Model and basic equations}

We consider the BD theory with matter fields;

$$
\hat{S}=\int d^{4} x \sqrt{-\hat{g}}\left[\frac{\phi}{2 \kappa^{2}} \hat{R}-\frac{\omega}{2 \kappa^{2} \phi}(\hat{\nabla} \phi)^{2}+\hat{L}_{m}\right],
$$


where $\kappa^{2}:=8 \pi$ and $\hat{L}_{m}$ is a matter Lagrangian. $\omega$ and $\phi$ are the BD parameter and the BD scalar field, respectively. We consider a "SU(2)" Proca field (a massive counterpart of the Yang-Mills field) as a matter field:

$$
\hat{L}_{m}=-\frac{1}{16 \pi g_{c}^{2}} \operatorname{Tr} \boldsymbol{F}^{2}-\frac{\mu^{2}}{8 \pi g_{c}^{2}} \operatorname{Tr} \boldsymbol{A}^{2}
$$

where $g_{c}$ and $\mu$ are the coupling constant and the mass of the Proca field, respectively. $\boldsymbol{F}$ is the field strength expressed by its potential $\boldsymbol{A}$ as $\boldsymbol{F}=d \boldsymbol{A}+\boldsymbol{A} \backslash \boldsymbol{A}$. We assume the spherically symmetric metric as

$$
d \hat{s}^{2}=-\hat{f}(\hat{r}, t) e^{-2 \hat{\delta}(\hat{r}, t)} d t^{2}+\hat{f}(\hat{r}, t)^{-1} d \hat{r}^{2}+\hat{r}^{2} d \Omega^{2},
$$

where $\hat{f}(\hat{r}, t)=1-2 \hat{m}(\hat{r}, t) / \hat{r}$, and the potential of the Proca field as ${ }^{1}$

$$
\boldsymbol{A}=[1+w(\hat{r}, t)]\left(-\boldsymbol{\tau}_{\phi} d \theta+\boldsymbol{\tau}_{\theta} \sin \theta d \phi\right),
$$

where $\tau_{\phi}$ and $\tau_{\theta}$ are the generators of su(2) Lie algebra.

We can examine this system also in the Einstein frame by a conformal transformation $g_{a b}=\phi \hat{g}_{a b}$ [12]. The equivalent action in the Einstein frame is

$$
S=\int d^{4} x \sqrt{-g}\left[\frac{R}{2 \kappa^{2}}-\frac{1}{2}(\nabla \varphi)^{2}+L_{m}\right],
$$

where

$$
\begin{gathered}
L_{m}=-\frac{1}{16 \pi g_{c}^{2}} \operatorname{Tr} \boldsymbol{F}^{2}-\frac{\mu^{2}}{8 \pi g_{c}^{2}} e^{-\kappa \beta \varphi} \operatorname{Tr} \boldsymbol{A}^{2}, \\
\beta:=\sqrt{\frac{2}{2 \omega+3}}, \quad \varphi:=\frac{1}{\kappa \beta} \ln \phi .
\end{gathered}
$$

With the area radius $r$, the metric becomes

$$
d s^{2}=-f(r, t) e^{-2 \delta(r, t)} d t^{2}+f(r, t)^{-1} d r^{2}+r^{2} d \Omega^{2},
$$

where $f(r, t)=1-2 m(r, t) / r$. We define dimensionless parameters as

$$
\bar{\mu}:=\mu / g_{c}, \quad \lambda_{h}:=r_{h} g_{c} .
$$

$r_{h}$ is the radius of the event horizon. For the numerical calculation, we introduce the following dimensionless variables: $\bar{t}:=t / r_{h}, \bar{r}:=r / r_{h}, \bar{m}:=m / r_{h}$. Then we obtain the basic equations in the Einstein frame;

\footnotetext{
${ }^{1}$ If the Yang-Mills field is massless and when we consider black hole solutions with the Yang-Mills field under the static and spherically symmetric Ansatz, we can impose this form without loss of generality for the gravitational sector. In the present case, however, we can just show that this is consistent with the field equation.
}

$$
\begin{gathered}
-\left(e^{\delta} f^{-1} \dot{w}\right)+\left(e^{-\delta} f w^{\prime}\right)^{\prime}+\frac{e^{-\delta}}{r^{2}}\left(1-w^{2}\right) w \\
-\lambda_{h}^{2} \mu^{2} e^{-\delta} e^{-\beta \varphi}(1+w)=0, \\
-\left(e^{\delta} f^{-1} \dot{\varphi}\right)+\frac{1}{r^{2}}\left(e^{-\delta} f r^{2} \varphi^{\prime}\right)^{\prime}+2 \mu^{2} \beta e^{-\delta} e^{-\beta \varphi} \\
\times \frac{(1+w)^{2}}{r^{2}}=0, \\
m^{\prime}=\frac{r^{2}}{4}\left(e^{2 \delta} f^{-1} \dot{\varphi}^{2}+f \varphi^{\prime 2}\right)+\frac{1}{\lambda_{h}^{2}}\left[e^{2 \delta} f^{-1} \dot{w}^{2}+f w^{\prime 2}\right. \\
\left.+\frac{\left(1-w^{2}\right)^{2}}{2 r^{2}}\right]+\mu^{2} e^{-\beta \varphi}(1+w)^{2}, \\
\delta^{\prime}=-\frac{r}{2}\left(e^{2 \delta} f^{-2} \dot{\varphi}^{2}+\varphi^{\prime 2}\right)-\frac{2}{\lambda_{h}^{2} r}\left(e^{2 \delta} f^{-2} \dot{w}^{2}+w^{\prime 2}\right), \\
\dot{m}=\frac{r^{2}}{2} f\left(\dot{\varphi} \varphi^{\prime}+\frac{4 \dot{w} w^{\prime}}{\lambda_{h}^{2} r^{2}}\right) .
\end{gathered}
$$

Here, we have omitted the bar on the variables. A prime and a dot denote the differentiations $\partial / \partial r$ and $\partial / \partial t$, respectively.

Since we will study the stability by the perturbation analysis, we expand the variables around a static solution as

$$
f(r, t)=f_{0}(r)+f_{1}(r, t) \epsilon+\frac{f_{2}(r, t)}{2} \epsilon^{2}+\cdots .
$$

Variables with the lower index 0 represent the static solution. The lower indices $i$ represent the $i$ th order of the perturbations. Then we obtain the perturbation equations order by order.

\section{B. Static solutions}

As for the static solution, we require the following boundary conditions. To satisfy asymptotic flatness, we impose

$$
m_{0}(\infty)=M, \quad \delta_{0}(\infty)=\varphi_{0}(\infty)=0, \quad w_{0}(\infty)=-1 .
$$

$M$ is the gravitational mass in the Einstein frame. For the existence of a regular horizon $r_{h}$, we impose

$$
m_{0}\left(r_{h}\right)=\frac{r_{h}}{2}, \quad \delta_{0}\left(r_{h}\right)<\infty, \quad \varphi_{0}\left(r_{h}\right)<\infty .
$$

$\delta_{0}\left(r_{h}\right), \varphi_{0}\left(r_{h}\right)$, and $w_{0}\left(r_{h}\right)$ are determined to satisfy the conditions (16) by integrating the field equations to infinity.

We describe the relation between the variables in the Einstein frame and those in the BD frame. At the horizon radius, it is easily found from Eq. (8) as 


$$
r_{h}=\sqrt{\phi\left(\hat{r}_{h}\right)} \hat{r}_{h}
$$

The gravitational mass in the BD frame $\hat{M}$ is defined by the time-time component of the metric (3) as ${ }^{2}$

$$
\hat{M}:=\hat{m}_{0}(\infty)+\lim _{\hat{r} \rightarrow \infty}\left(\hat{r} \hat{\delta}_{0}\right) .
$$

It satisfies the relation

$$
\hat{M}=M+M_{s},
$$

where $M_{s}$ is a scalar mass defined by the asymptotic form of the scalar field $e^{\kappa \beta \varphi_{0}} \sim 1+2 M_{s} / r$ as $r \rightarrow \infty$.

The static black hole solutions in this system are investigated in detail (see Ref. [11]). We summarize some of their properties which are useful for later discussions.

Let us consider the special case $\omega \rightarrow \infty$, which corresponds to general relativity. In the massless case, i.e., the ordinary Yang-Mills field, there are infinite number of solutions called colored black holes [13], which are classified by the node number of the Yang-Mills potential $w$, for fixed horizon radius. Since the Yang-Mills potential decays faster than $r^{-1}$, the colored black holes have no global color charge. It is formed by the delicate balance between the gravitational force and the gauge force. If we add the mass term in the vector field, another type of solutions appears, which is the Schwarzschild counterpart in the massless limit. Hence it is considered as the structure of the self-gravitating Proca field around the Schwarzschild black hole. The solution with large node number and/or the large horizon radius disappears. We concentrate on the solutions with one node for matters of convenience, where Schwarzschild type solution is stable and the colored black hole type has one unstable mode against the linear perturbation [14].

For the finite value of $\omega$, we also find two types of solutions. Without the mass term, the matter Lagrangean is invariant under the conformal transformation in 4-dimension. So the colored black hole solution is the same as one in general relativity. We show the relation between the gravitational mass $\hat{M}$ and the horizon radius $\hat{r}_{h}$ in the BD frame in Fig. 1. There is no black hole solution above the critical horizon radius (which corresponds to point $A$ in Fig. 1), since the nontrivial structure of the Proca field which spreads out to the Compton wavelength $(\sim 1 / \mu)$ is swallowed into the horizon as the black hole becomes large, resulting in a Schwarzschild space-time.

We can find the cusp structure at the point $C$ in general relativity, which is a symptom of stability change in the catastrophe theoretical point of view. We can infer from the entropy consideration that the solutions in the branch shown by the solid line are stable and those in the dotted branch are unstable [5]. On the contrary, the cusp structure disappears in BD theory. If we consider the analogy with the case in gen-

\footnotetext{
${ }^{2}$ For the gravitational mass in the Einstein frame, there is no such ambiguity because $\delta_{0}$ in the Einstein frame decays faster than $\sim 1 / r$.
}

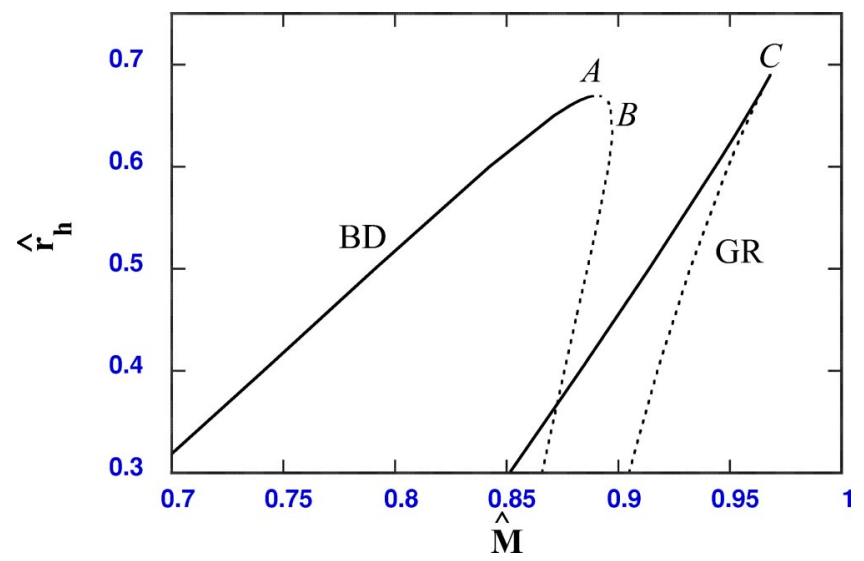

FIG. 1. $\hat{M}-\hat{r}_{h}$ diagram in the BD frame. The mass of the Proca field is $\mu=0.15 g_{c} m_{p}$ and the BD parameter is $\omega=0$.

eral relativity, stability change may occur at point $A$ (the maximum point of $\hat{r}_{h}$ ) or at point $B$ (the maximum point of $\hat{M})$.

If we see the $M-r_{h}$ diagram in the Einstein frame (Fig. 2), we can find cusp structures in both theories. Point $A$ in Fig. 1 corresponds to the cusp. Hence we may interpret that the solid branch is stable while the dotted branch is unstable even in BD theory. We should confirm it.

We discussed in Ref. [11] that the nonexistence of the cusp structure in the BD frame is due to the fact that the variables $\hat{M}$ and $\hat{r}_{h}$ are inappropriate to the catastrophe variables. Since stability should be independent of the frame one chooses, it is natural to choose frame independent variables as catastrophe variables. We develop this idea in the following sections.

\section{Linear perturbation analysis}

We perform a linear perturbation analysis to clarify whether stability judgement by catastrophe theory in the Einstein frame is correct or not.

By defining the new variable $\psi_{1}:=r \varphi_{1}$ and substituting,

$$
\psi_{1}(r, t)=\xi(r) e^{i \sigma t},
$$

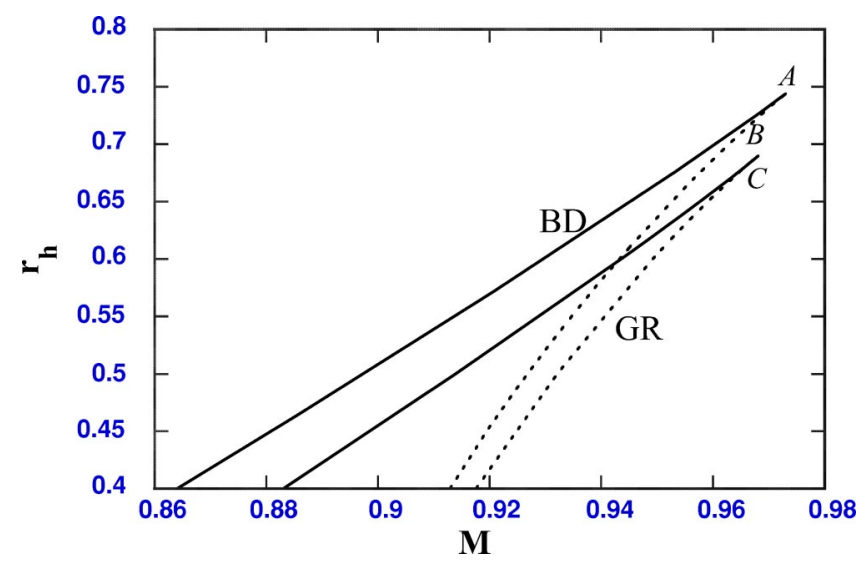

FIG. 2. $M-r_{h}$ diagram in the Einstein frame corresponding to the solutions in Fig. 1. 


$$
\begin{aligned}
& w_{1}(r, t)=\eta(r) e^{i \sigma t}, \\
& m_{1}(r, t)=\zeta(r) e^{i \sigma t},
\end{aligned}
$$

we find the eigenequation

$$
\frac{d^{2}}{d r^{* 2}}\left(\begin{array}{l}
\xi \\
\eta
\end{array}\right)-e^{-2 \delta_{0}} f_{0}\left[\begin{array}{ll}
U_{11} & U_{12} \\
U_{21} & U_{22}
\end{array}\right]\left(\begin{array}{l}
\xi \\
\eta
\end{array}\right)=-\sigma^{2}\left(\begin{array}{l}
\xi \\
\eta
\end{array}\right),
$$

where we have used the tortoise coordinate $r^{*}$ defined by

$$
\frac{d r}{d r^{*}}=e^{-\delta_{0}} f_{0}
$$

The components of the potential matrix are

$$
\begin{aligned}
U_{11}= & -\frac{1}{r}\left(f_{0} \delta_{0}^{\prime}+\frac{2 m_{0}^{\prime}}{r}-\frac{2 m_{0}}{r^{2}}\right)+2 \mu^{2} \beta e^{-\beta \varphi_{0}} \\
& \times \frac{\left(1+w_{0}\right)^{2}}{r^{2}}\left(\beta-r \varphi_{0}^{\prime}\right)-\frac{B r}{2} f_{0} \varphi_{0}^{\prime}, \\
U_{12}= & -\frac{4\left(1-w_{0}^{2}\right) w_{0} \varphi_{0}^{\prime}}{\lambda_{h}^{2} r^{2}}-4 \mu^{2} e^{-\beta \varphi_{0}} \frac{1+w_{0}}{r}(\beta \\
& \left.-r \varphi_{0}^{\prime}\right)-\frac{4 B}{2 \lambda_{h}^{2}} f_{0} w_{0}^{\prime}, \\
U_{21}= & -\mu^{2} \beta e^{-\beta \varphi_{0}} \frac{1+w_{0}}{r}\left[\frac{2}{r}\left(1+w_{0}\right) w_{0}^{\prime}+\lambda_{h}^{2}\right] \\
& -\frac{A}{2} r f_{0} \varphi_{0}^{\prime}, \quad \frac{1-3 w_{0}^{2}}{r^{2}}+\lambda_{h}^{2} \mu^{2} e^{-\beta \varphi_{0}-\frac{2}{\lambda_{h}^{2}} A f_{0} w_{0}^{\prime},} \\
U_{22}= & -\frac{4 w_{0}^{\prime}}{r}\left[\frac{w_{0}\left(1-w_{0}^{2}\right)}{\lambda_{h}^{2} r^{2}}-\mu^{2} e^{-\beta \varphi_{0}}\left(1+w_{0}\right)\right] \\
& \left.-\frac{1}{r}\right)
\end{aligned}
$$

where

$$
\begin{aligned}
& A:=w_{0}^{\prime}\left(\varphi_{0}^{\prime 2}+\frac{4 w_{0}^{\prime 2}}{\lambda_{h}^{2} r^{2}}\right)+\frac{2}{r}\left(\delta_{0}^{\prime} w_{0}^{\prime}+\frac{w_{0}^{\prime}}{r}-w_{0}^{\prime \prime}\right), \\
& B:=2\left(\delta_{0}^{\prime} \varphi_{0}^{\prime}-\frac{\varphi_{0}^{\prime}}{r}-\varphi_{0}^{\prime \prime}\right)+r \varphi_{0}^{\prime}\left(\varphi_{0}^{\prime 2}+\frac{4 w_{0}^{\prime 2}}{\lambda_{h}^{2} r^{2}}\right) .
\end{aligned}
$$

$\zeta$ is obtained by the linear combination of $\eta$ and $\xi$ as

$$
\zeta=\frac{r}{2} f_{0} \varphi_{0}^{\prime} \xi+\frac{2}{\lambda_{h}^{2}} f_{0} w_{0}^{\prime} \eta
$$

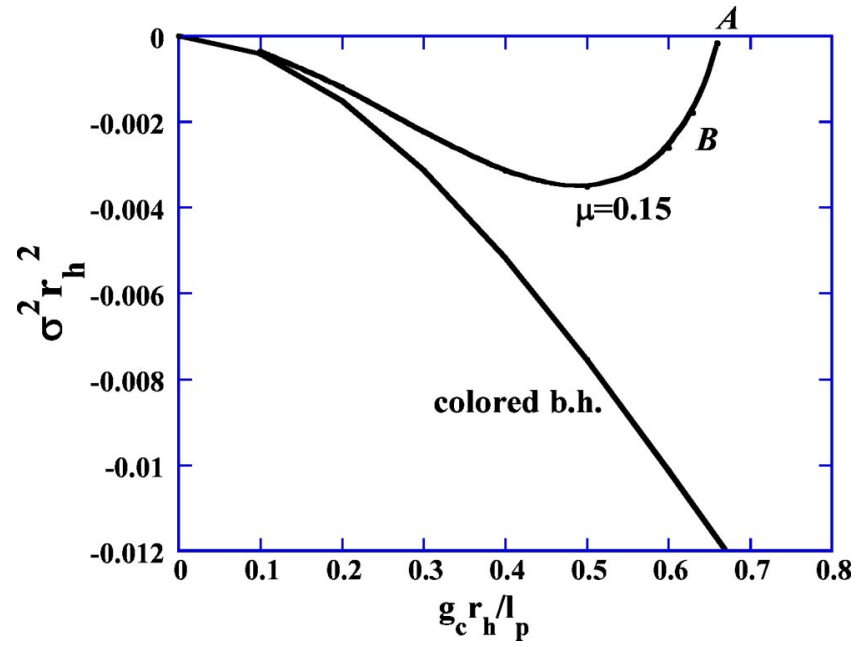

FIG. 3. $r_{h}-\sigma^{2}$ diagram for Proca black holes $(\mu=0.15)$ in BD theory $(\omega=0)$ and colored black holes for reference. Point $A$ corresponds to point $A$ in Fig. 1 .

If there is at least one mode with negative eigenvalue $\sigma^{2}<0$, the solution is unstable. For a colored black hole, we find one unstable mode [15]. We show its eigenvalue in Fig. 3. As for the Proca black hole in BD theory, we find an unstable mode for the solutions in the dotted branch in Figs. 1 and 2. Points $A$ and $B$ in Fig. 3 correspond to those in Figs. 1 and 2. Thus we can confirm that the catastrophe analysis in Einstein frame gives consistent results with the linear perturbation analysis.

\section{CATASTROPHE VARIABLES IN GENERALIZED THEORIES OF GRAVITY}

Here, we explain how to apply catastrophe theory to black hole stability along the same lines as in Ref. [5] and try to extend it to generalized theories of gravity.

If we consider compact stars, such as a neutron star, binding energy plays a role as a natural potential function of catastrophe theory [16]. After the collapse to black holes, much information of the stellar object is lost as is stated in black hole no-hair conjecture. Hence we cannot calculate binding energy. Kerr-Newman black holes are characterized by three parameters, which are regarded as hairs. We know these hairs are stable. However, for exotic black holes which have other hairs it is not trivial whether they are stable or not. Actually there are many exotic black holes with unstable hairs [17]. When we examine their stability by catastrophe theory, we should first determine which variables we adopt. This is a nontrivial subject.

We proposed the stability analysis of the black holes in general relativity via catastrophe theory in previous papers. If we use the variables which are related to the first law of black hole thermodynamics, we can obtain appropriate results. We briefly explain its relation to catastrophe theory. We describe the potential function of the system by $F(s, x)$, where $s$ and $x$ are a control parameter and a state variable, respectively. The equilibrium point of the system is written as 


$$
\frac{\partial F(s, x)}{\partial x}=0
$$

We write $x$ which satisfies this condition as $x_{e q}(s)$. We also define $F_{e q}(s):=F\left[s, x_{e q}(s)\right]$. Then we obtain

$$
\begin{gathered}
\frac{d F_{e q}(s)}{d s}=\left(\frac{\partial F}{\partial s}\right)_{e q}+\left(\frac{\partial F}{\partial x}\right)_{e q} \frac{d x_{e q}}{d s}=\left(\frac{\partial F}{\partial s}\right)_{e q}, \\
\frac{d^{2} F_{e q}(s)}{d s^{2}}=\left(\frac{\partial^{2} F}{\partial s^{2}}\right)_{e q}+\left(\frac{\partial^{2} F}{\partial x \partial s}\right)_{e q} \frac{d x_{e q}}{d s} .
\end{gathered}
$$

Because of the condition that $F_{e q}(s)$ is an equilibrium point, we also have

$$
0=\frac{d}{d s}\left(\frac{\partial F}{\partial x}\right)_{e q}=\left(\frac{\partial^{2} F}{\partial x \partial s}\right)_{e q}+\left(\frac{\partial^{2} F}{\partial x^{2}}\right)_{e q} \frac{d x_{e q}}{d s}
$$

If we eliminate $d x_{e q} / d s$ from Eq. (35) by using this equation, we obtain

$$
\frac{d^{2} F_{e q}(s)}{d s^{2}}=\left(\frac{\partial^{2} F}{\partial s^{2}}\right)_{e q}-\left(\frac{\partial^{2} F}{\partial x \partial s}\right)_{e q}^{2} /\left(\frac{\partial^{2} F}{\partial x^{2}}\right)_{e q} .
$$

Since the point where stability changes corresponds to the inflection point of the potential function, we have

$$
\left(\frac{\partial^{2} F}{\partial x^{2}}\right)_{e q}=0
$$

Then, if

$$
\left(\frac{\partial^{2} F}{\partial x \partial s}\right)_{e q}=0
$$

is not satisfied, we have

$$
\frac{d^{2} F_{e q}(s)}{d s^{2}}=\infty
$$

In the previous papers, we regard a horizon radius $r_{h}$ or equivalently black hole entropy $S$ as a potential function $F$ and the gravitational mass $M$ as a control parameter $s$. If we assume the first law of black hole thermodynamics, $d F_{e q} / d s$ corresponds to $d S / d M=1 / T$. Because of Eq. (39), stability changes at

$$
\frac{d(1 / T)}{d M}=\infty
$$

We show the $\hat{M}-1 / T$ (Fig. 4) and $M-1 / T$ (Fig. 5) corresponding to the solutions in Fig. 1. These diagrams also show that stability changes at points $A$ and $C$ and suggest that thermodynamic variables which satisfy the first law is useful. Similar discussion can be seen in Ref. [18]. Note that there is no contradiction even if we regard $M$ as a potential function and

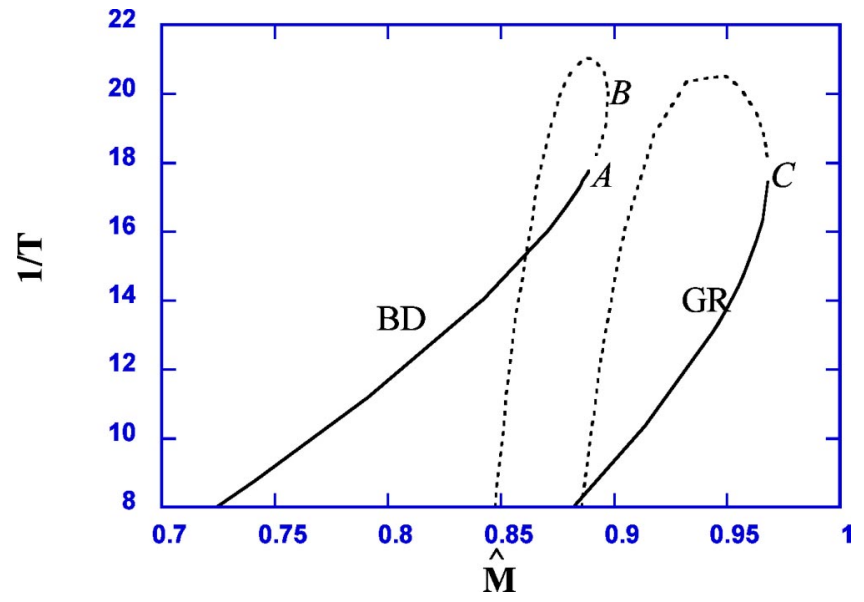

FIG. 4. $\hat{M}-1 / T$ diagram for the solutions corresponding to Fig. 1.

$S$ as a control parameter for black holes at present, since stability change can be characterized by $d T / d S=\infty$ in this case and it gives the same result. (You may find it by considering Fig. 2 and by viewing Fig. 5 upside down.) When the matter fields have parameters, for example, the mass of the Proca field $\mu$, they also become other control parameters. We assume that the field strength at horizon $B_{h}$ or the lapse function at horizon $\delta_{h}$ is a state variable. To verify choosing $B_{h}$ or $\delta_{h}$ as a state variable and the condition (38) are beyond our scope at present.

Next, we explain the necessary condition for $M$ to be a potential function. We show a schematic diagram of $M-B_{h}-S$ of the Proca black hole in general relativity in Fig. 6. In this diagram, the mass of the Proca field, which may be one of the control parameters, is fixed. The extremum of the entropy corresponds to the cusp on the $M-S$ projected plane (see Fig. 1). When we fix the entropy of the black hole, we expect the potential curve in Fig. 7. The points $S$ and $U$ correspond to a stable and an unstable solution, respectively. Other points correspond to the nonequilibrium solution. The potential curve for each $M$ forms a so-called Whitney surface. The bifurcation set, which is a set of singular points of a catas-

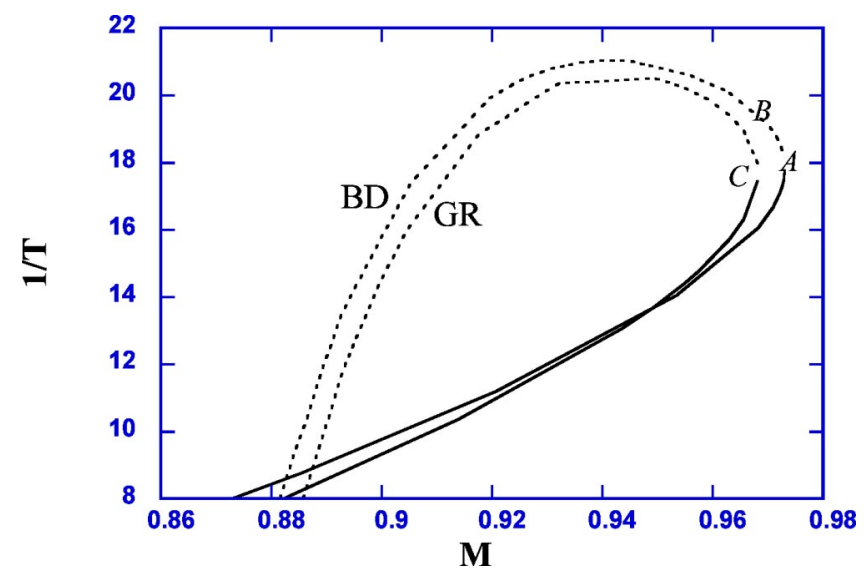

FIG. 5. $M-1 / T$ diagram for the solutions corresponding to Fig. 1. 


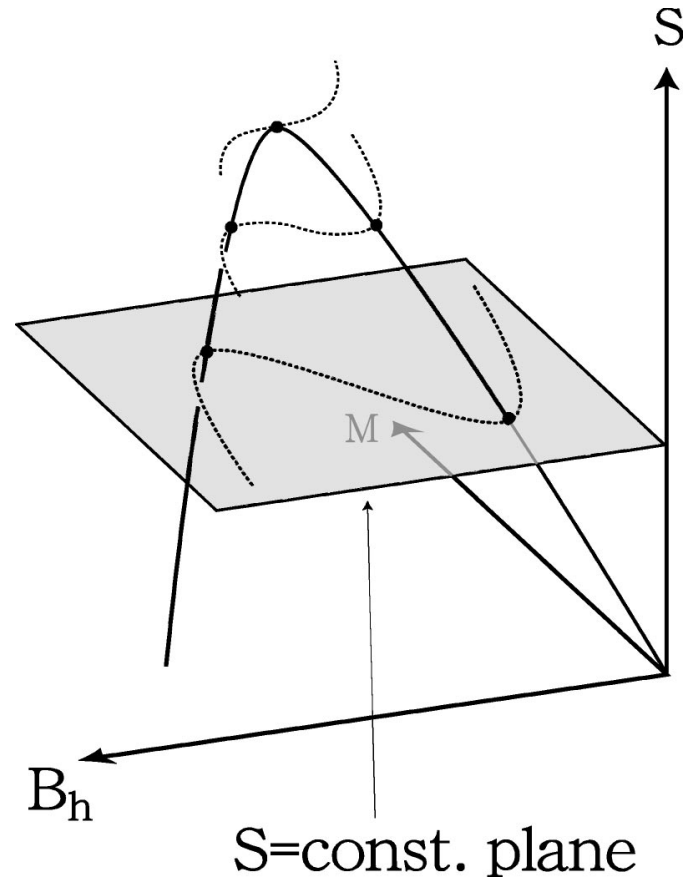

FIG. 6. $M-B_{h}-S$ diagram for Proca black hole. In this diagram, static solutions are denoted in bold lines.

trophe map, becomes a simple curve on the control plane $M-\mu$. Thus we can interpret this system as a fold catastrophe.

What we consider in this paper is whether catastrophe theory is also applicable for the black holes in generalized theories of gravity or not, and if it is, what variables are appropriate for the catastrophe variables. First, we should note that there is an ambiguity in which frame do we consider. Actually, as we showed in Sec. II, the gravitational

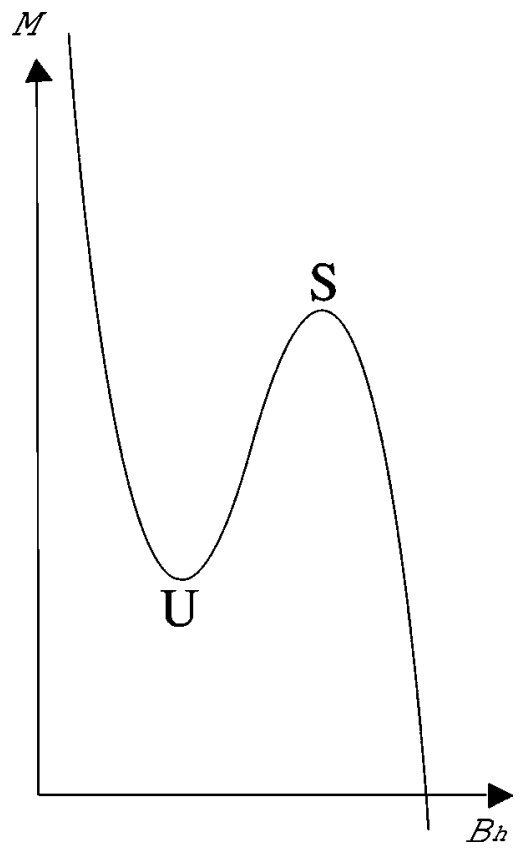

FIG. 7. $B_{h}-M$ diagram for Proca black holes, when $\mu$ and $r_{h}$ are fixed. mass and the area radius depend on the frame in BD theory.

In the previous section we have got a clue that a cusp structure appears and the results obtained by linear perturbation analysis exactly coincide with those in catastrophe theory if we choose the variables in the Einstein frame. We cannot say immediately, however, that the variables in the Einstein frame are appropriate for catastrophe theory or the others are, not even if there appears the cusp structure since it can be always created at any point we like by choosing other inappropriate variables.

Since the stability of black holes does not depend on the frame, it would be favorable to choose conformally invariant variables. The second clue is the thermodynamic variables proposed by Iyer and Wald which satisfy the first law of black hole thermodynamics in generalized theories of gravity [7]. These variables have remarkable properties in that they are invariant under the field redefinitions [19] and the conformal transformation [20]. Hence these variables seem suitable for the analysis by catastrophe theory. Furthermore, these thermodynamic variables coincide with those in the Einstein frame when gravitational theories can be transformed to the Einstein-Hilbert action by the conformal transformation. Hence we naturally expect that these thermodynamic variables are suitable for catastrophe theory.

In the following section, we show that $M$ satisfies one of the important conditions as a potential function by drawing the local Whitney surface around the static solutions. Thereby we use the linear perturbation analysis. If we fix the entropy, the local minima (maxima) of the thermodynamic mass correspond to the linearly stable (unstable) solution. Since we fix the horizon radius in the Einstein frame, which is equivalent to fixing entropy, and perturb the fields in linear perturbation analysis, the latter correspondence is easy to be verified. Thus, as a first step to justify the analysis by catastrophe theory by using the thermodynamic variables in the generalized theories of gravity, we first transform to the Einstein frame and show

$$
\begin{gathered}
m_{1}(\infty)=: M_{1}=0, \\
\sigma^{2}>0 \Leftrightarrow m_{2}(\infty)=: M_{2}>0 .
\end{gathered}
$$

These are important conditions to say that the dynamical stability can be judged via catastrophe theory. Similar discussion about the gravitational mass is also found in Ref. [21], although the relation with catastrophe theory is not pointed out.

\section{LINEAR PERTURBATION AND LOCAL WHITNEY SURFACE}

Here, we show the properties which should be satisfied for the thermodynamic mass to be a potential function discussed in the previous section. As for the metric, we assume Eq. (8). Since we consider in the Einstein frame, the gravitational part of the action is the ordinary Einstein-Hilbert action 


$$
S=\int d^{4} x \sqrt{-g}\left[\frac{R}{2 \kappa^{2}}+L_{m}\right]
$$

The matter action $L_{m}$ should be considered as the conformal transformed action. We assume the following conditions: (i) zeroth order variables of $L_{m}$ are independent of $\delta$. (ii) $\delta^{\prime}$ does not depend on the matter field $w$ itself explicitly (it can depend on the derivative of $w$ ). This means that we include a scalar field and a peculiar component of the Yang-Mills potential [e.g., $w$ in Eq. (4)]. Here we consider a single matter field, it is straightforward to generalize to multifields cases. These Ansätze seem rather technical, but they cover a large class of models and most of the exotic black hole solutions in static spherically symmetric space-time discovered previously satisfy these Ansätze.

We consider the reduced action using the metric functions. Varying the reduced action with respect to $\delta$ and $m$, we obtain

$$
\begin{aligned}
& m^{\prime}=-\frac{\kappa^{2}}{2} r^{2} L_{m}, \\
& \delta^{\prime}=-\frac{\kappa^{2}}{2} r^{2} \partial_{m} L_{m},
\end{aligned}
$$

where $\partial_{f}:=\partial / \partial f$. As for the matter field equation, we obtain

$$
\left(\partial_{w} \boldsymbol{L}\right)^{\prime}+\left(\partial_{w^{\prime}} \boldsymbol{L}\right)^{\prime}-\partial_{w} \boldsymbol{L}=0,
$$

where $\boldsymbol{L}:=\sqrt{-g} L_{m}$. The zeroth order equation becomes

$$
\left(\partial_{w^{\prime}} \boldsymbol{L}\right)^{\prime}-\partial_{w} \boldsymbol{L}=0 .
$$

Next, we consider the first order equations. From Eq. (44), the equation for the first order of the mass function $m_{1}$ is

$$
\begin{aligned}
\left(e^{\left.-\delta_{0} m_{1}\right)^{\prime}}\right. & =-\frac{\kappa^{2} r^{2} e^{-\delta_{0}}}{2 \sqrt{-g_{0}}}\left(w_{1}^{\prime} \partial_{w^{\prime}} \boldsymbol{L}_{\boldsymbol{0}}+w_{1} \partial_{w} \boldsymbol{L}_{\boldsymbol{0}}\right) \\
& =-\frac{\kappa^{2}}{2 \sin \theta}\left(w_{1} \partial_{w^{\prime}} \boldsymbol{L}_{\boldsymbol{0}}\right)^{\prime}
\end{aligned}
$$

where we have used Eq. (47). Using the fact that $m_{1}$ is constant at the horizon, the final form is

$$
m_{1}=-\frac{\kappa^{2} r^{2}}{2} w_{1} \partial_{w^{\prime}} L_{m}
$$

By using the fact that $w_{0}$ decays faster than $1 / r$ at $r \rightarrow \infty$ because of the asymptotically flatness and the renormalizability of $w_{1}$, we obtain $M_{1}=0$, i.e., the relation (41).

From Eq. (45), the equation for the first order lapse function $\delta_{1}$ is

$$
\delta_{1}^{\prime}=-\frac{\kappa^{2} r^{2}}{2 \sqrt{-g_{0}}}\left(w_{1}^{\prime} \partial_{w^{\prime}} \partial_{m} \boldsymbol{L}_{\boldsymbol{0}}+m_{1} \partial_{m}^{2} \boldsymbol{L}_{\boldsymbol{0}}\right),
$$

where we use assumptions (i) and (ii). Using Eqs. (46) and (47), the first order equation for the field variable $w$ is

$$
\begin{gathered}
\ddot{w}_{1} \partial_{w^{\prime}}^{2} \boldsymbol{L}_{\boldsymbol{0}}+\left(w_{1}^{\prime} \partial_{w^{\prime}}^{2} \boldsymbol{L}_{\boldsymbol{0}}\right)^{\prime}+\left(\partial_{w} \partial_{w^{\prime}} \boldsymbol{L}_{\boldsymbol{0}}\right)^{\prime} w_{1}-w_{1} \partial_{w}^{2} \boldsymbol{L}_{\boldsymbol{0}} \\
+\left(\partial_{m} \partial_{w^{\prime}} \boldsymbol{L}_{\boldsymbol{0}}\right)^{\prime} m_{1}+m_{1}^{\prime} \partial_{m} \partial_{w^{\prime}} \boldsymbol{L}_{\boldsymbol{0}}+\delta_{1}^{\prime} \partial_{\delta} \partial_{w^{\prime}} \boldsymbol{L}_{\boldsymbol{0}} \\
-m_{1} \partial_{m} \partial_{w^{\prime}} \boldsymbol{L}_{\boldsymbol{0}}=0 .
\end{gathered}
$$

Eliminating $m_{1}, m_{1}^{\prime}$, and $\delta_{1}^{\prime}$ using Eqs. (48) and (50), this equation becomes

$$
\ddot{w}_{1}-\frac{V}{\left(r^{2} \sin \theta\right)^{2}}\left[V w_{1}^{\prime}\right]^{\prime}-U(r) w_{1}=0,
$$

where

$$
\begin{gathered}
U(r):=-\left(\partial_{\dot{w}}^{2} \boldsymbol{L}_{\boldsymbol{0}}\right)^{-1}\left[\left(\partial_{w^{\prime}} \partial_{w^{\prime}} \boldsymbol{L}_{\boldsymbol{0}}\right)^{\prime}-\partial_{w}^{2} \boldsymbol{L}_{\boldsymbol{0}}\right. \\
-\frac{\boldsymbol{\kappa}^{2}}{2 \sin \theta} \partial_{m} \partial_{w^{\prime}} \boldsymbol{L}_{\boldsymbol{0}}\left(e^{\delta_{0}} \partial_{w^{\prime}} \boldsymbol{L}_{\boldsymbol{0}}\right)^{\prime} \\
\left.-\frac{\boldsymbol{\kappa}^{4}}{4 \sin ^{2} \theta} e^{2 \delta_{0}} \partial_{m}^{2} \boldsymbol{L}_{\boldsymbol{0}}\left(\partial_{w^{\prime}} \boldsymbol{L}_{\boldsymbol{0}}\right)^{2}\right] \\
V:=\partial_{w^{\prime}}^{2} \boldsymbol{L}_{\boldsymbol{0}} .
\end{gathered}
$$

If we introduce the tortoise coordinate

$$
\partial / \partial r^{*}:=\frac{-V}{r^{2} \sin \theta} \frac{\partial}{\partial r}
$$

Eq. (52) is written as

$$
\ddot{w}_{1}-\frac{1}{r^{2}}\left(r^{2} \frac{\partial^{2} w_{1}}{\partial r^{* 2}}\right)-U(r) w_{1}=0 .
$$

If we put the form of separation of variable as

$$
w_{1}=\eta(r) e^{i \sigma t}
$$

Eq. (55) becomes

$$
\frac{1}{r^{2}}\left(r^{2} \frac{\partial^{2} \eta}{\partial r^{* 2}}\right)+U \eta=-\sigma^{2} \eta
$$

$\sigma^{2}$ is the eigenvalue.

On the other hand, the second order equation of the mass function $m_{2}$ is

$$
\begin{aligned}
& e^{-\delta_{0}}\left(m_{2}^{\prime}-\delta_{0}^{\prime} m_{2}\right) \\
&=-\frac{\kappa^{2} r^{2}}{2} \frac{e^{-\delta_{0}}}{\sqrt{-g}}\left[\left|w_{1}\right|^{2} \partial_{w}^{2}+\left|w_{1}^{\prime}\right|^{2} \partial_{w^{\prime}}^{2}+\left|\dot{w}_{1}\right|^{2} \partial_{w}^{2}\right. \\
&+\left|m_{1}\right|^{2} \partial_{m}^{2}+2 \operatorname{Re}\left(w_{1}^{*} w_{1}^{\prime}\right) \partial_{w^{\prime}} \partial_{w^{\prime}}+2 \operatorname{Re}\left(w_{1}^{*} m_{1}\right) \partial_{w} \partial_{m} \\
&\left.+2 \operatorname{Re}\left(w_{1}^{\prime} * m_{1}\right) \partial_{m} \partial_{w^{\prime}}\right] \boldsymbol{L}_{\boldsymbol{0}} .
\end{aligned}
$$


We can integrate this as

$$
\begin{aligned}
{\left[e^{\left.-\delta_{0} m_{2}\right]_{r_{h}}^{\infty}=}\right.} & -\frac{\kappa^{2}}{2 \sin \theta} \int_{r_{h}}^{\infty} d r\left\{\left|\dot{w}_{1}\right|^{2} \partial_{w^{2}}^{2} \boldsymbol{L}_{\boldsymbol{0}}+\left|w_{1}^{\prime}\right|^{2} \partial_{w^{\prime}}^{2} \boldsymbol{L}_{\boldsymbol{0}}\right. \\
& +\left[\partial_{w}^{2} \boldsymbol{L}_{\boldsymbol{0}}-\left(\partial_{w^{\prime}} \partial_{w^{\prime}} \boldsymbol{L}_{\boldsymbol{0}}\right)^{\prime}\right. \\
& +\frac{\kappa^{2}}{2 \sin \theta} \partial_{m} \partial_{w^{\prime}} \boldsymbol{L}_{\boldsymbol{0}}\left(e^{\left.\delta_{0} \partial_{w^{\prime}} \boldsymbol{L}_{\boldsymbol{0}}\right)^{\prime}}\right. \\
& \left.\left.+\frac{\boldsymbol{\kappa}^{4}}{4 \sin ^{2} \theta} e^{2 \delta_{0}} \partial_{m}^{2} \boldsymbol{L}_{\boldsymbol{0}}\left(\partial_{w^{\prime}} \boldsymbol{L}_{\boldsymbol{0}}\right)^{2}\right]\left|w_{1}\right|^{2}\right\}
\end{aligned}
$$

Using assumption (ii) and Eq. (53), we finally obtain

$$
\begin{aligned}
M_{2}= & -\frac{\kappa^{2}}{2 \sin \theta} \int_{r_{h}}^{\infty} d r\left[\left|\dot{w}_{1}\right|^{2} \partial_{w}^{2} \boldsymbol{L}_{\mathbf{0}}+\left|w_{1}^{\prime}\right|^{2} \partial_{w^{\prime}}^{2} \boldsymbol{L}_{\boldsymbol{0}}\right. \\
& -U(r)\left|w_{1}\right|^{2} \partial_{\left.w_{w}^{2} \boldsymbol{L}_{\mathbf{0}}\right],} \\
= & \frac{\kappa^{2} \sigma^{2}}{2} \int_{-\infty}^{\infty} d r^{*} r^{2} \eta^{2} .
\end{aligned}
$$

The right-hand side is positive definite except for the trivial one $\eta=0$. Hence we can satisfy Eq. (42). Equation (60) says that when we draw the Whitney surface by using the thermodynamical variables, it becomes ridge (trough) around the stable (unstable) solutions. The static solution with 0 -mode just coincides with the inflection point (i.e., $\partial^{2} S / \partial B_{H}^{2}=0$ ) of the Whitney surface. Hence we can obtain important correspondence between the linear analysis and catastrophe theory by using the thermodynamic variables defined by Iyer and Wald. It is worth noting that if we perform corresponding analysis in the action (1), the gravitational mass $\hat{M}$ in the BD frame does not satisfy Eqs. (41) and (42). Thus if we discuss stability via catastrophe theory in the theory including a scalar field such as the Brans-Dicke field or the dilaton field, care should be taken.

\section{CONCLUSION}

In this paper, we investigated stability analysis of black holes via a catastrophe theory and its relation to the analysis by linear perturbation analysis. As a concrete model, we first considered the Proca black hole in BD theory and found that we can analyze stability via catastrophe theory by using the variables in the Einstein frame, which are related to the thermodynamic variables defined in Ref. [7]. We also confirmed stability by linear perturbation, and it is consistent with the analysis via catastrophe theory. Based on this observation, we considered black holes with general matter fields in gravitational theories that can be transformed to the EinsteinHilbert action by conformal transformation. We obtained the result that thermodynamic mass does play a potential function, which suggests that the thermodynamic variables play important roles in catastrophic analysis. This consideration will be also helpful to understand the stability of general $\mathrm{D} p$-brane solutions as discussed in Ref. [4].

Our next task is to refine our discussion and to generalize the result to other gravitational theories. Actually, if we apply catastrophe theory by using thermodynamic variables defined in Ref. [7] for a string effective action which includes the Gauss-Bonnet term and a dilaton field [22-24], the results are consistent with the analysis by linear perturbation [25]. We also have concerns about whether or not generalized entropy satisfies the second law of black hole thermodynamics. Investigating these properties may shed new light on what the black hole thermodynamics is.

\section{ACKNOWLEDGMENTS}

Special thanks to J. Koga and T. Tachizawa for useful discussions. This work was supported by the Waseda University Grant for Special Research Projects.
[1] A. Strominger and C. Vafa, Phys. Lett. B 379, 99 (1996); J.M. Maldacena and A. Strominger, Phys. Rev. Lett. 77, 428 (1996).

[2] J.M. Maldacena, Adv. Theor. Math. Phys. 2, 231 (1998); E. Witten, ibid. 2, 253 (1998).

[3] S.S. Gubser and I. Mitra, J. High Energy Phys. 08, 018 (2001).

[4] H.S. Reall, Phys. Rev. D 64, 044005 (2001).

[5] K. Maeda, T. Tachizawa, T. Torii, and T. Maki, Phys. Rev. Lett. 72, 450 (1994); T. Torii, K. Maeda, and T. Tachizawa, Phys. Rev. D 51, 1510 (1995); T. Tachizawa, K. Maeda, and T. Torii, ibid. 51, 4054 (1995).

[6] A. Chamblin, R. Emparan, C.V. Johnson, and R.C. Myers, Phys. Rev. D 60, 064018 (1999).

[7] R.M. Wald, Phys. Rev. D 48, 3427 (1993); V. Iyer and R.M. Wald, ibid. 50, 846 (1994); 52, 4430 (1995).

[8] G. Kang, Phys. Rev. D 54, 7483 (1996).

[9] T. Poston and I. Stewart, Catastrophe Theory and Its Applications (Pitman, London, 1978); R. Thom, Strucure Stability and Morphogenesis (Benjamin, New York, 1975).
[10] C. Brans and R.H. Dicke, Phys. Rev. 124, 925 (1961).

[11] T. Tamaki, K. Maeda, and T. Torii, Phys. Rev. D 57, 4870 (1998); 60, 104049 (1999).

[12] R.H. Dicke, Phys. Rev. 125, 2163 (1962).

[13] P. Bizon, Acta Phys. Pol. B 25, 877 (1994).

[14] B.R. Greene, S.D. Mathur, and C.M. O’Neill, Phys. Rev. D 47, 2242 (1993).

[15] M.S. Volkov, O. Brodbeck, G. Lavrelashvili, and N. Straumann, Phys. Lett. B 349, 3438 (1995).

[16] For review, see, e.g., S. L. Shapiro and S. A. Teukolsky, Black Holes, White Dwarfs, and Neutron Stars (Wiley, New York, 1983).

[17] As a review paper see K. Maeda, J. Korean Phys. Soc. 28, S468 (1995); and M.S. Volkov and D.V. Gal'tsov, Phys. Rep. 319, 1 (1999).

[18] O. Kaburaki, I. Okamoto, and J. Katz, Phys. Rev. D 47, 2234 (1993); J. Katz, I. Okamoto, and O. Kaburaki, Class. Quantum Grav. 10, 1323 (1993). 
[19] T. Jacobson, G. Kang, and R.C. Myers, Phys. Rev. D 49, 6587 (1994).

[20] J. Koga and K. Maeda, Phys. Rev. D 58, 064020 (1998).

[21] O. Brodbeck, M. Heusler, and N. Straumann, Phys. Rev. D 53, 754 (1996).

[22] T. Torii, H. Yajima, and K. Maeda, Phys. Rev. D 55, 739 (1997).
[23] E.E. Donets and D.V. Gal'tsov, Phys. Lett. B 302, 411 (1993); 312, 391 (1993).

[24] P. Kanti, N.E. Mavromatos, J. Rizos, K. Tamvakis, and E. Winstanely, Phys. Rev. D 54, 5049 (1996); 57, 6255 (1998); P. Kanti and K. Tamvakis, Phys. Lett. B 392, 30 (1997).

[25] T. Torii and K. Maeda, Phys. Rev. D 58, 084004 (1998). 